\title{
Direct optical detection
}

\section{Günter Gauglitz • Jiř́i Homola}

Published online: 15 March 2015

(C) Springer-Verlag Berlin Heidelberg 2015

Over the last 20 years, optical methods have been increasingly used in analytical and bioanalytical applications. These methods have been applied in two rather broad ways: for direct and for indirect detection. Whereas direct-detection approaches typically enable the detection of an analyte in a single step, indirect approaches usually require the use of labels. The most commonly used indirect-detection approach is based on the use of fluorescent labels, via measuring the fluorescence emitted by the label either attached to the analyte or to a molecule that binds to the analyte. Although fluorescence-based approaches enable detection and quantification of analytes at extremely low concentrations, the use of labels increases the complexity of the method and imposes limitations on the systems that can be studied using fluorescence. Conversely, direct label-

Published in the topical collection Direct Optical Detection with guest editors Guenter Gauglitz and Jiri Homola.

G. Gauglitz $(\bowtie)$

Institute of Physical and Theoretical Chemistry, Eberhard Karls Universität, Auf der Morgenstelle 18, 72076 Tübingen, Germany

e-mail: abc.gg@ipc.uni-tuebingen.de

\section{J. Homola}

Institute of Photonics and Electronics ASCR, Chaberska 57,

Prague 8 18251, Czech Republic

e-mail: homola@ufe.cz free approaches have been revealed to be able to overcome some of these limitations and expand the use of optical methods to new fields of application, for example, biomolecular-interaction analysis.

Direct label-free detection of chemical and biological analytes takes advantage of a large and diverse group of optical methods. Absorption spectroscopy detects analytes by measuring the absorption of light at selected wavelengths that match the analytes' absorption bands. In Raman spectroscopy, analytes are detected on the basis of their specific spectral Raman-scattering fingerprints. Recently, optical methods based on the measurement of analyte-induced refractive index changes have attracted a great deal of attention, especially for affinity biosensing. These methods measure refractive index changes produced by the binding of analyte molecules to special biorecognition molecules (e.g. antibodies, nucleic acids, peptides), which are immobilized on a surface of an optical transducer and are able to recognize and capture analyte molecules. The transducers that are able to measure analyte-induced refractive index changes are mainly based on the following methods: interferometry (Young and Mach-Zehnder integrated optical interferometers, Fabry-Pérot interferometer), spectroscopy of guided modes of optical waveguides (resonant mirror sensor, grating coupler sensor, surface plasmon resonance sensor), and optical resonators (microsphere resonator sensor, ring resonator 
sensor). Most of these transducers use an evanescent field to observe biomolecular interactions. As in other fields of analysis, imaging has also gained a growing share in biosensor-based bioanalysis, and numerous direct optical approaches have been expanded to imaging modalities (e.g. surface plasmon resonance, white light interferometry). Typical applications of this detection technology include high throughput screening of pharmaceutical libraries, screening of peptide binders against target proteins, receptor-peptide interactions, and even cell receptor morphology screening.

Direct label-free detection has gone beyond applications in research, and is attracting an increasing amount of interest from industry. For instance, the U.S. Food and Drug Administration (FDA) increasingly requires both the conditions for obtaining label-free interaction data and the testing conditions of pharmaceuticals to be as close as possible to in vivo (with a large variety of assays). Thus, the range of applications of label-free detection methods is no longer only focussed on small molecules and therapeutic antibodies, but also includes proteomics and target and non-target screening. It has been realized that by using such methods, more complex assays and more complex biomolecular interactions can be examined. Therapeutic drug monitoring, effect-based analysis, analyte-cell interaction, and even signalling pathways are examples of important upcoming applications. Accordingly, as well as special congresses on label-free detection technology, sessions dealing with direct optical biomolecular-interaction analysis are also scheduled for other bioanalysis congresses.

This topical collection contains both reviews and original research articles covering different aspects of direct optical sensing, from technological developments to new applications. It also serves to reflect the breadth of the field and to illustrate the potential of direct optical detection technology.

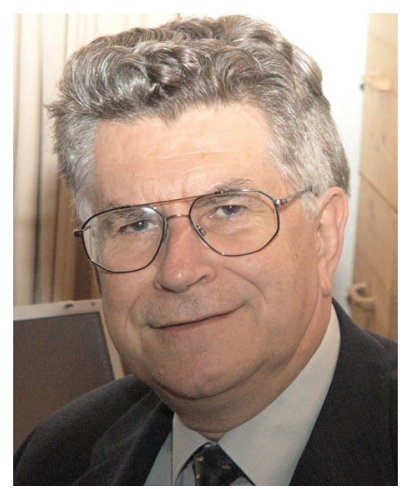

Günter Gauglitz is Senior Professor at the Eberhard-Karls University of Tübingen working in analytical and physical chemistry. He was chairman of the GDCh Division of Analytical Chemistry and chaired the Europt(r)ode VIII meeting. For more than 15 years his main scientific interests have centered on research and development in chemical and biochemical sensors, with special focus on the characterization of interfaces of polymers and biomembrane surfaces, spectroscopy techniques, use of spectral interferometry to monitor changes in optical thickness of thin layers, and effects of Fresnel reflectivity at interfaces. He has been Editor of Analytical and Bioanalytical Chemistry (ABC) since 2002 .

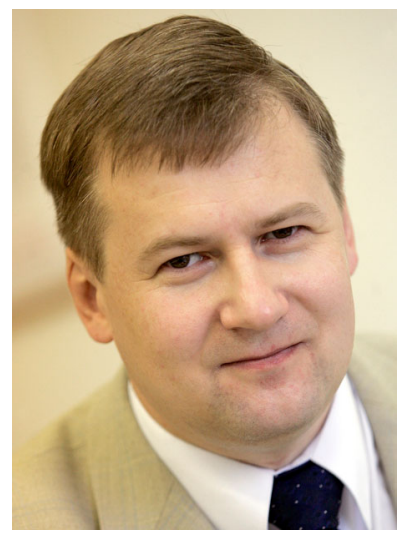

Jiří Homola is Director of the Institute of Photonics and Electronics of the Czech Academy of Sciences (Prague, Czech Republic). $\mathrm{He}$ is also Professor at Charles University in Prague (Czech Republic) and Affiliate Professor at the University of Washington, Seattle (USA). His research interests are in photonics and biophotonics, with emphasis on optical sensors and biosensors. $\mathrm{He}$ is a member of the editorial boards of Sensors and Actuators B (Elsevier) and Journal of Sensors (Hindawi) and a member of the international advisory board of Analytical and Bioanalytical Chemistry (Springer). He is a member of the permanent steering committees of Advanced Study Course on Optical Chemical Sensors and Europt(r)ode Conference Series. He has received several awards, including the Award of the Minister of Education, Youth and Sports for Outstanding Research, Experimental Development and Innovation, Czech Head Award, Otto Wichterle Prize, and Roche Diagnostics Prize for Sensor Technology. He has edited two books, written seven book chapters, and over 130 papers in peerreviewed scientific journals. 